CALT-68-2527

hep-th/0410282

\title{
Quantum string integrability and AdS/CFT
}

\author{
Ian Swanson \\ California Institute of Technology \\ Pasadena, CA 91125, USA
}

\begin{abstract}
Recent explorations of the AdS/CFT correspondence have unveiled integrable structures underlying both planar $\mathcal{N}=4$ super-Yang-Mills theory and type IIB string theory on $A d S_{5} \times S^{5}$. Integrability in the gauge theory emerges from the fact that the dilatation generator can be identified with the Hamiltonian of an integrable quantum spin chain, and the classical string theory has been shown to contain infinite towers of hidden currents, a typical signature of integrability. Efforts to match the integrable structures of various classical string configurations to those of corresponding gauge theory quantum spin chains have been largely successful. By studying a semiclassical expansion about a class of point-like solitonic solutions to the classical string equations of motion on $A d S_{5} \times S^{5}$, we take a step toward demonstrating that integrability in the string theory survives quantum corrections beyond tree level. Quantum fluctuations are chosen to align with background curvature corrections to the pp-wave limit of $A d S_{5} \times S^{5}$, and we present evidence for an infinite tower of local bosonic charges that are conserved by the quantum theory to quartic order in the expansion. We explicitly compute several higher charges based on a Lax representation of the worldsheet sigma model and provide a prescription for matching the eigenvalue spectra of these charges with corresponding quantities descending from the integrable structure of the gauge theory.
\end{abstract}

December 18, 2013 


\section{Introduction}

The emergence of integrable structures from planar $\mathcal{N}=4$ super-Yang-Mills (SYM) theory and type IIB string theory on $A d S_{5} \times S^{5}$ has renewed hope that 't Hooft's formulation of large- $N_{c}$ QCD may eventually lead to an exact solution. If both the gauge and string theories are in fact integrable, each will admit infinite towers of hidden charges and, analogous to the usual identification of the string theory Hamiltonian with the gauge theory dilatation generator, there will be an infinite number of mappings between the higher hidden charges of both theories. This has led to many novel tests of the AdS/CFT correspondence, particularly in the context of the pp-wave/BMN limits [1, 2, 3]. Barring an explicit solution, one would hope that both theories will at least be shown to admit identical Bethe ansatz equations, allowing us to explore a much larger region of the gauge/string duality.

The fact that the gauge theory harbors integrable structures was realized by Minahan and Zarembo when they discovered that a particular $S O(6)$-invariant sector of the SYM dilatation generator can be mapped, at one-loop order in the 't Hooft coupling $\lambda=g_{\mathrm{YM}}^{2} N_{c}$, to the Hamiltonian of an integrable quantum spin chain with $S O(6)$ vector lattice sites [4]. The Hamiltonian of this system can be diagonalized by solving a set of algebraic Bethe ansatz equations: the problem of computing operator anomalous dimensions in this sector of the gauge theory was thus reduced in [4] to solving the set of Bethe equations specific to the $\mathfrak{s o}(6)$ sector of the theory. The correspondence between operator dimensions and integrable spin-chain systems at one loop in $\lambda$ was extended to include the complete $\mathfrak{p s u}(2,2 \mid 4)$ superconformal symmetry algebra of planar $\mathcal{N}=4 \mathrm{SYM}$ theory by Beisert and Staudacher in 5. Studies of higher-loop integrability in the gauge theory were advanced in [6, 7, where so-called long-range Bethe ansatz equations, which are understood to encode interactions on the spin lattice that extend beyond nearest-neighbor sites, were developed for a closed bosonic $\mathfrak{s u}(2)$ sector of the gauge theory. In this context, closure refers to the fact that operators in this sector are guaranteed by group theoretical constraints to not mix with other operators in the theory under the action of the dilatation generator (to all orders in $\lambda$ ) [8, 9], and such sectors are typically labeled by the subalgebra of the full superconformal symmetry algebra under which they are invariant. (The spectral predictions provided by the long-range ansatz of [7] were checked against an alternative virial technique in [10, and agreement was obtained to a high degree of precision.) The dynamics of the gauge theory therefore appear to be copasetic with the expectations of integrability, at least to three-loop order in the 't Hooft expansion, and there is convincing evidence that this extends to even higher order [7, 11].

Concurrent with the introduction of the Bethe ansatz formalism in the $\mathfrak{s o}(6)$ sector of the gauge theory [4], related developments emerged from studies of semiclassical configurations of rotating string on $A d S_{5} \times S^{5}$. This branch of investigation began with [12], where the pp-wave limit of the string theory was reinterpreted in the context of a semiclassical expansion about certain solitonic solutions in the full $A d S_{5} \times S^{5}$ target space. Using this semiclassical picture, Frolov and Tseytlin computed a class of two-spin string solutions in [13, demonstrating explicitly how stringy corrections in the large-spin limit give rise to systems that can be 
understood as generalizations of the original pp-wave solution studied in [1, 2, 3. This work was extended by a more general study of multi-spin string solutions in [14, where the authors provided a detailed prescription for making direct comparisons with perturbative gauge theory. (For a more complete review of the development and current status of semiclassical string theory and the match-up with gauge theory, see [15] and references therein.) Early indications of integrability in the classical limit of the string theory emerged when it was shown that a certain configuration of the Green-Schwarz superstring action on $A d S_{5} \times S^{5}$ admits an infinite set of classically conserved non-local charges, and may therefore be an integrable theory itself [16] (see also [17] for a reduction to the pp-wave system). The gauge theory analogue of this non-local symmetry was studied in [18, 19], where a direct connection with the string analysis was made to one-loop order in $\lambda$. Various subtleties surrounding studies of the non-local (or Yangian) algebra arise at higher loops, and further work is certainly warranted.

In addition to the sector of non-local charges, however, integrable systems typically admit an infinite tower of local, mutually commuting charges, each of which are diagonalized by a set of Bethe equations [20, 21]. The presence of such a sector of hidden, classically conserved bosonic charges in the string theory was pointed out in [22]. Moreover, in accordance with the expectations of AdS/CFT duality, various studies have been successful in matching hidden local charges in the classical string theory to corresponding quantities in the quantum spinchain formulation of $\mathcal{N}=4$ SYM theory. In [23, for example, Arutyunov and Staudacher constructed an infinite series of conserved local charges in the bosonic string theory by solving the Bäcklund equations associated with certain extended classical solutions of the $O(6)$ string sigma model. The local charges generated by the Bäcklund transformations were then matched to corresponding conserved charges obtained from an integrable quantum spin chain on the gauge theory side. In fact, they were able to demonstrate agreement between both sides of the duality for the entire infinite tower of local commuting charges. This study was extended in [24, 25], where it was shown that a general class of rotating classical string solutions can be mapped to solutions of a Neumann (or Neumann-Rosochatius) integrable system. More recently, a class of three-spin classical string solutions was shown in [26] to generate hidden local charges (again via Bäcklund transformations) that match their gauge theory counterparts to one-loop order. (For a thorough review of the match-up of semiclassical string integrable structures with corresponding structures in the gauge theory, see also [11, 15].)

The mapping between string and gauge theory integrable structures was studied from a somewhat different perspective in [27, where it was shown that the generator of local, classically conserved currents in the string theory is related in certain sectors to a particular Riemann-Hilbert problem which is reproduced precisely by the gauge theory integrable structure at one and two loops in $\lambda$. An analogous treatment of the corresponding RiemannHilbert problem in non-compact sectors of the gauge/string duality was carried out in [28], and an extension of these studies to a larger $\mathfrak{s o}(6)$ sector was recently achieved in [29]. The structure of the higher-loop Riemann-Hilbert problem descending from the classical string theory and its relationship with the corresponding gauge theory problem was used in con- 
junction with the long-range gauge theory Bethe ansatz of [7] to develop an ansatz which, albeit conjecturally, is purported to interpolate between the classical and quantum regimes of the string theory 30. Although this proposal is not a proof of quantum integrability on the string side, it was demonstrated in 31] that the quantized string theory in the near-ppwave limit yields a general multi-impurity spectrum that matches the string Bethe ansatz spectrum of [30. The intricacy of this match-up is quite remarkable, and stands as strong evidence that this ansatz is correct for the string theory, at least to $O(1 / J)$ in the large angular momentum (or background curvature) expansion. Furthermore, the proposed string Bethe equations can accommodate the strong-coupling $\lambda^{1 / 4}$ scaling behavior predicted in 32. The spin chain theory implied by these Bethe equations, however, appears to disagree with that of the gauge theory, even at weak coupling 33 .

Although the Bethe equations of [30] reproduce several predictions of the string theory in a highly nontrivial way, a direct test of quantum integrability (beyond tree level) in the string theory is still needed: this is the intent of the present work. Early steps in this direction were taken in 34, where the presence of a conserved local charge responsible for a certain parity degeneracy in the near-pp-wave string spectrum was examined at sixth-order in field fluctuations, or at $O\left(1 / J^{2}\right)$ in the large- $J$ expansion. Various subtleties of the analysis (possibly involving the proper renormalization of the theory at $O\left(1 / J^{2}\right)$ in the expansion) made it difficult to reach any concrete conclusions, however. In this paper we take a more immediate approach, relying primarily on a Lax representation of the classical string sigma model and studying a semiclassical expansion about certain point-like solitonic solutions. The goal is to establish the existence of a series of conserved, mutually commuting charges in the string theory that can be quantized and studied using first-order perturbation theory. By aligning field fluctuations with the finite-radius curvature expansion in [35, 36, 37, 38, we are able to study quantum corrections to quartic order, or to one loop beyond tree level. We show directly that several of the low-lying hidden charges in the series are conserved by the quantum theory to this order in the expansion, and we propose a method for matching specific eigenvalues of these charges to corresponding spectral quantities in the gauge theory.

The paper is organized as follows. In Section 2 we review the procedure for string quantization in the near-pp-wave limit developed in [36, 37], and we demonstrate how background curvature corrections to the pp-wave theory can be interpreted as quantum corrections in a particular semiclassical expansion about point-like classical string solutions. In Section 3 we show how a Lax representation of the $O(4,2) \times O(6)$ nonlinear sigma model can be modified to encode the string dynamics to the order of interest in this semiclassical expansion. We then generate a series of hidden local charges by expanding a perturbed monodromy matrix of the Lax representation in powers of the spectral parameter. In section 4 we compute the eigenvalues of these charges in certain protected subsectors of the theory in the space of two-impurity string states. The resulting spectra are then compared on the $S^{5}$ subspace with those of corresponding charges descending from the $\mathfrak{s u}(2)$ integrable sector of the gauge theory. We provide a prescription for matching the spectra of local charges on both sides of the duality, and carry out this matching procedure to eighth order in the spectral parameter. To the extent that they can be compared reliably, the gauge and string theory predictions 
are shown to match to this order (and presumably continue to agree at higher orders). We are thus led to believe that the integrable structure of the classical string theory survives quantization, at least to the first subleading order in field fluctuations beyond tree level. We conclude in the final section by outlining future directions of study.

\section{Semiclassical string quantization in $A d S_{5} \times S^{5}$}

Most of the literature comparing semiclassical bosonic string theory in $A d S_{5} \times S^{5}$ to corresponding sectors of gauge theory operators has focused on classical extended string solutions to the worldsheet sigma model in either "folded" or "circular" configurations, where certain components of the string angular momentum (ie. certain charges of the Cartan subalgebra of the global symmetry group) are taken to be large (see, eg. [13, 14, 24]). The latter amounts to choosing a so-called "spinning ansatz" for the string configuration [13, 14, 15, 22, 24, 25], and solutions endowed with such an ansatz can be identified with periodic solutions of the Neumann (or Neumann-Rosochatius) integrable system. The standard bosonic worldsheet action is usually chosen with flat worldsheet metric so that it is easily rewritten in terms of $\mathbf{R}^{6}$ embedding coordinates and identified with an $O(4,2) \times O(6)$ sigma model. In the present study we will modify this treatment to allow for curvature corrections to the worldsheet metric, a complication that we are forced to confront when moving beyond tree level in lightcone gauge [36, 37.

We begin with a particular form of the $A d S_{5} \times S^{5}$ target space metric, chosen originally in [36, 37] for the fact that it admits a simple form for the spin connection:

$$
d s_{A d S_{5} \times S^{5}}^{2}=R^{2}\left[-\left(\frac{1+\frac{1}{4} z^{2}}{1-\frac{1}{4} z^{2}}\right)^{2} d t^{2}+\left(\frac{1-\frac{1}{4} y^{2}}{1+\frac{1}{4} y^{2}}\right)^{2} d \phi^{2}+\frac{d z_{k} d z_{k}}{\left(1-\frac{1}{4} z^{2}\right)^{2}}+\frac{d y_{k^{\prime}} d y_{k^{\prime}}}{\left(1+\frac{1}{4} y^{2}\right)^{2}}\right] .
$$

While we will not address fermions in this study, we will eventually return to the crucial issues of supersymmetry, and the metric choice in eqn. (2.1) will undoubtedly simplify further investigations. Here, the bosonic coordinates $z_{k}$ and $y_{k^{\prime}}$ span a transverse $S O(4) \times S O(4)$ space, with $j, k, l, \ldots \in 1, \ldots, 4$ and $j^{\prime}, k^{\prime}, l^{\prime}, \ldots \in 5, \ldots, 8$. The $z_{k}$ coordinates are always chosen to lie in the $A d S_{5}$ subspace, while $y_{k^{\prime}}$ are coordinates on $S^{5}$. The scale factor $R$ is the common radius of both subspaces. By defining

$$
\cosh \rho \equiv \frac{1+\frac{1}{4} z^{2}}{1-\frac{1}{4} z^{2}} \quad \cos \theta \equiv \frac{1-\frac{1}{4} y^{2}}{1+\frac{1}{4} y^{2}}
$$

we may write the $\mathbf{R}^{6} \times \mathbf{R}^{6}$ embedding coordinates of $A d S_{5}$ and $S^{5}$ as

$$
\begin{array}{ll}
Z_{k}=\sinh \rho \frac{z_{k}}{\|z\|} & Z_{0}+i Z_{5}=\cosh \rho e^{i t}, \\
Y_{k^{\prime}}=\sin \theta \frac{y_{k^{\prime}}}{\|y\|} & Y_{5}+i Y_{6}=\cos \theta e^{i \phi}
\end{array}
$$


with $\|z\| \equiv \sqrt{z_{k} z_{k}}$. The coordinates $Z_{P}$, with $P, Q=0, \ldots, 5$, parameterize $A d S_{5}$ and are contracted over repeated indices using the metric $\eta_{P Q}=(-1,1,1,1,1,-1)$. The coordinates $Y_{M}$, with $M, N=1, \ldots, 6$, encode the $S^{5}$ geometry, and are contracted with a Euclidean metric.

Decomposing the theory into $A d S_{5}$ and $S^{5}$ subspaces, the usual conformal-gauge worldsheet action

$$
S=-\int d^{2} \sigma h^{a b} G_{\mu \nu} \partial_{a} x^{\mu} \partial_{b} x^{\nu}
$$

can be written as

$$
\begin{aligned}
S & =\int d^{2} \sigma\left(\mathcal{L}_{A d S_{5}}+\mathcal{L}_{S^{5}}\right) \\
\mathcal{L}_{A d S_{5}} & =-\frac{1}{2} h^{a b} \eta_{P Q} \partial_{a} Z_{P} \partial_{b} Z_{Q}+\frac{\tilde{\varphi}}{2}\left(\eta_{P Q} Z_{P} Z_{Q}+1\right) \\
\mathcal{L}_{S^{5}} & =-\frac{1}{2} h^{a b} \partial_{a} Y_{M} \partial_{b} Y_{M}+\frac{\varphi}{2}\left(Y_{M} Y_{M}-1\right) .
\end{aligned}
$$

The quantities $\varphi$ and $\tilde{\varphi}$ act as Lagrange multipliers in the action, enforcing the following conditions: ${ }^{1}$

$$
\eta_{P Q} Z_{P} Z_{Q}=-1, \quad Y_{M} Y_{M}=1
$$

The action in eqn. (2.4) must also be supplemented by the standard conformal gauge constraints, and the worldsheet metric $h^{a b}$ (the worldsheet indices run over $a, b \in \tau, \sigma$ ) will be allowed to acquire curvature corrections in accordance with these constraints.

We wish to study a semiclassical expansion about the following classical point-like (or "BMN-like") solutions to the sigma model equations of motion:

$$
t=\phi=p_{-} \tau \quad z_{k}=y_{k}=0
$$

The expansion is defined in terms of quantum field fluctuations according to the following rescaling prescription:

$$
t \rightarrow x^{+} \quad \phi \rightarrow x^{+}+\frac{x^{-}}{\sqrt{\xi}} \quad z_{k} \rightarrow \frac{z_{k}}{\xi^{1 / 4}} \quad y_{k} \rightarrow \frac{y_{k}}{\xi^{1 / 4}}
$$

(A similar but notably different choice was made in [13].) This particular choice of lightcone coordinates will allow us to maintain a constant momentum distribution on the worldsheet. Additionally, as noted in [36, 37, it will have the effect of eliminating all normal-ordering ambiguities from the resulting worldsheet theory, an outcome which is particularly desirable in the present study. Furthermore, we note that if we identify $\xi \equiv R^{4}$, the proposed expansion

\footnotetext{
${ }^{1}$ Note that, in general, $\varphi$ and $\tilde{\varphi}$ will depend on dynamical variables. We thank Arkady Tseytlin for clarification on this point.
} 
about the classical solution in eqn. (2.8) is identical to the large-radius curvature expansion about the pp-wave limit of $A d S_{5} \times S^{5}$ studied in [36, 37, 38. In other words, we have chosen a perturbation to the classical point-like string geodesic that reproduces the target-space curvature perturbation to the pp-wave limit. The background metric in eqn. (2.1) thus yields the following large- $R$ expansion:

$$
\begin{aligned}
d s^{2}= & 2 d x^{+} d x^{-}-\left(x^{A}\right)^{2}\left(d x^{+}\right)^{2}+\left(d x^{A}\right)^{2} \\
& +\frac{1}{R^{2}}\left[-2 y^{2} d x^{+} d x^{-}+\frac{1}{2}\left(y^{4}-z^{4}\right)\left(d x^{+}\right)^{2}+\left(d x^{-}\right)^{2}+\frac{1}{2} z^{2} d z^{2}-\frac{1}{2} y^{2} d y^{2}\right] \\
& +O\left(R^{-4}\right),
\end{aligned}
$$

where the pp-wave geometry emerges at leading order, and $x^{A}$ are transverse $S O(8)$ coordinates, with $A \in 1, \ldots, 8$.

The details of quantizing the string Hamiltonian in this setting are given in [36, 37] (see also [39, 40, 41, 42, 43, for further details), though we will briefly review the salient points here. The lightcone Hamiltonian $H_{\mathrm{LC}}$ is the generator of worldsheet time translations, and is defined in terms of the Lagrangian by

$$
-H_{L C}=-p_{+}=\delta \mathcal{L} / \delta \dot{x}^{+}
$$

(or $\Delta-J$ in the language of BMN), and this variation is performed prior to any gauge fixing. The non-physical lightcone variables $x^{ \pm}$are removed from the Hamiltonian by fixing lightcone gauge $x^{+}=p_{-} \tau$ and replacing $x^{-}$with dynamical variables by enforcing the conformal gauge constraints

$$
T_{a b}=\frac{\delta \mathcal{L}}{\delta h^{a b}}=0
$$

This procedure can be defined order-by-order in the large- $R$ expansion. At leading order, for example, we obtain the following from eqn. (2.12):

$$
\begin{aligned}
\dot{x}^{-} & =\frac{p_{-}}{2}\left(x^{A}\right)^{2}-\frac{1}{2 p_{-}}\left[\left(\dot{x}^{A}\right)^{2}+\left(x^{A}\right)^{2}\right]+O\left(1 / R^{2}\right), \\
x^{\prime-} & =-\frac{1}{p_{-}} \dot{x}^{A} x^{\prime A}+O\left(1 / R^{2}\right) .
\end{aligned}
$$

The conformal gauge constraints themselves are only consistent with the equations of motion if the worldsheet metric acquires curvature corrections (ie. $h$ departs from the flat metric $h=\operatorname{diag}(-1,1)$ ), which we express symbolically as $\tilde{h}^{a b}$ according to

$$
h=\left(\begin{array}{cc}
-1+\tilde{h}^{\tau \tau} / R^{2} & \tilde{h}^{\tau \sigma} / R^{2} \\
\tilde{h}^{\tau \sigma} / R^{2} & 1+\tilde{h}^{\sigma \sigma} / R^{2}
\end{array}\right) .
$$


The requirement that $\operatorname{det} h=-1$ implies $\tilde{h}^{\tau \tau}=\tilde{h}^{\sigma \sigma}$ and, for future reference, the correction terms $\tilde{h}^{a b}$ are given explicitly to the order of interest by

$$
\begin{aligned}
& \tilde{h}^{\tau \tau}=\frac{1}{2}\left(z^{2}-y^{2}\right)-\frac{1}{2 p_{-}^{2}}\left[\left(\dot{x}^{A}\right)^{2}+\left(x^{\prime}\right)^{2}\right], \\
& \tilde{h}^{\tau \sigma}=\frac{1}{p_{-}^{2}} \dot{x}^{A} x^{\prime A} .
\end{aligned}
$$

Finally, we note that the canonical momenta associated with the physical worldsheet excitations, defined by the variation $p_{A}=\delta \mathcal{L} / \delta x^{A}$, also acquire $O\left(1 / R^{2}\right)$ corrections: consistent quantization requires that these corrections be taken into account. Expressed in terms of canonical variables, the final bosonic Hamiltonian takes the form

$$
\begin{aligned}
H_{\mathrm{LC}}= & \frac{p_{-}}{2 R^{2}}\left(x^{A}\right)^{2}+\frac{1}{2 p_{-} R^{2}}\left(\left(p_{A}\right)^{2}+\left(x^{\prime A}\right)^{2}\right) \\
& +\frac{1}{R^{4}}\left\{\frac{1}{4 p_{-}}\left[z^{2}\left(p_{y}^{2}+y^{\prime 2}+2 z^{\prime 2}\right)-y^{2}\left(p_{z}^{2}+z^{\prime 2}+2 y^{\prime 2}\right)\right]+\frac{p_{-}}{8}\left[\left(x^{A}\right)^{2}\right]^{2}\right. \\
- & \left.\frac{1}{8 p_{-}^{3}}\left\{\left[\left(p_{A}\right)^{2}\right]^{2}+2\left(p_{A}\right)^{2}\left(x^{\prime A}\right)^{2}+\left[\left(x^{A}\right)^{2}\right]^{2}\right\}+\frac{1}{2 p_{-}^{3}}\left(x^{\prime A} p_{A}\right)^{2}\right\}+O\left(1 / R^{6}\right),
\end{aligned}
$$

where the pp-wave Hamiltonian emerges as expected at leading order. The lightcone momentum $p_{-}$is identified (via the AdS/CFT dictionary) with the modified 't Hooft parameter $\lambda^{\prime}$ according to

$$
p_{-}=1 / \sqrt{\lambda^{\prime}}=J / \sqrt{\lambda}
$$

From the point of view of the semiclassical analysis, we are working to two-loop order in quantum corrections. Since the quadratic theory can be quantized exactly, however, we can study the quartic interaction Hamiltonian using standard first-order perturbation theory. A detailed analysis of the resulting spectrum of this perturbation can be found in [31, 36, 37, 38. In the course of those studies it was noticed that, analogous to the gauge theory closed sectors studied in [8, 9, 44, 45], certain sectors emerged from the string analysis that decouple from the remainder of the theory to all orders in $\lambda^{\prime}$. One sector, which maps to the $\mathfrak{s l}(2)$ sector of the gauge theory, is diagonalized by bosonic string states excited in the $A d S_{5}$ subspace and forming symmetric-traceless irreps in spacetime indices. The corresponding sector of symmetric-traceless $S^{5}$ string bosons maps to the closed $\mathfrak{s u}(2)$ sector in the gauge theory. The block-diagonalization of these sectors in the string Hamiltonian will be an important tool in the present analysis: just as all higher hidden local charges in the gauge theory are simultaneously diagonalized by a single Bethe ansatz, all of the higher hidden charges descending from the string theory should be block-diagonalized by these particular string states as well. 


\section{Lax representation}

The goal is to determine whether a ladder of higher local charges can be computed and quantized (albeit perturbatively), analogous to the existing treatment of the near-pp-wave Hamiltonian given in eqn. (2.16) above. To quartic order in the semiclassical expansion defined by eqn. (2.9), the difference between the string sigma model in eqns. (2.52.6) and that of the $O(4,2) \times O(6)$ sigma model, defined by

$$
\begin{aligned}
\mathcal{L}_{O(4,2)} & =-\frac{1}{2} \eta_{P Q} \partial_{a} Z_{P} \partial^{a} Z_{Q}+\frac{\tilde{\varphi}}{2}\left(\eta_{P Q} Z_{P} Z_{Q}+1\right) \\
\mathcal{L}_{O(6)} & =-\frac{1}{2} \partial_{a} Y_{M} \partial^{a} Y_{M}+\frac{\varphi}{2}\left(Y_{M} Y_{M}-1\right)
\end{aligned}
$$

will essentially amount to an interaction perturbation due to curvature corrections to the worldsheet metric. We therefore find it useful to rely on a known Lax representation of the $O(4,2) \times O(6)$ sigma model; this representation will define an unperturbed theory, and we will add perturbations by hand to recover the full interaction Hamiltonian in eqn. (2.16). (For a general introduction to the Lax methodology in integrable systems, the reader is referred to [21.) Since worldsheet curvature corrections only appear at $O\left(1 / R^{2}\right)$, the reduction to the $O(4,2) \times O(6)$ sigma model at leading order in the expansion will be automatic.

For simplicity, we start from the four-dimensional Lax representation given for the $O(6)$ sigma model in 25]. (see also [46] for details), and work only to leading order in the semiclassical expansion. The complexified coordinates

$$
\mathcal{Y}_{1}=Y_{1}+i Y_{2} \quad \mathcal{Y}_{2}=Y_{3}+i Y_{4} \quad \mathcal{Y}_{3}=Y_{5}+i Y_{6}
$$

are used to form a unitary matrix $S_{S^{5}}$

$$
S_{S^{5}}=\left(\begin{array}{cccc}
0 & \mathcal{Y}_{1} & -\mathcal{Y}_{2} & \overline{\mathcal{Y}}_{3} \\
-\mathcal{Y}_{1} & 0 & \mathcal{Y}_{3} & \overline{\mathcal{Y}}_{2} \\
\mathcal{Y}_{2} & -\mathcal{Y}_{3} & 0 & \overline{\mathcal{Y}}_{1} \\
-\overline{\mathcal{Y}}_{3} & -\overline{\mathcal{Y}}_{2} & -\overline{\mathcal{Y}}_{1} & 0
\end{array}\right)
$$

in terms of which one may form the following $S U(4)$-valued currents:

$$
A_{a}=S_{S^{5}} \partial_{a} S_{S^{5}}^{\dagger} \text {. }
$$

The equations of motion of the $O(6)$ sigma model

$$
\partial_{a} \partial^{a} Y_{M}+\varphi Y_{M}=0
$$

are then encoded by the auxiliary system of linear equations

$$
\left(\partial_{\sigma}-U\right) X=\left(\partial_{\tau}-V\right) X=0,
$$


where the Lax pair $U$ and $V$ are defined by

$$
U=\frac{1}{1+\gamma} A_{-}-\frac{1}{1-\gamma} A_{+}, \quad V=-\frac{1}{1+\gamma} A_{-}-\frac{1}{1-\gamma} A_{+} .
$$

The constant $\gamma$ is a free spectral parameter, and $A_{ \pm}$are defined by $A_{ \pm} \equiv \frac{1}{2}\left(A_{\tau} \pm A_{\sigma}\right)$. Note that on the $S O(4)$ subspace spanned by $y_{k^{\prime}}$, eqn. (3.5) reduces to the pp-wave equations of motion on $S^{5}$ :

$$
\ddot{y}_{k^{\prime}}-y_{k^{\prime}}^{\prime \prime}+p_{-}^{2} y_{k^{\prime}}=0 \text {. }
$$

The utility of the Lax representation arises from the fact that $U$ and $V$ may be considered as local connection coefficients, and a consistency equation for the auxiliary linear problem can be reinterpreted as a flatness condition for the $(U, V)$-connection:

$$
\partial_{\tau} U-\partial_{\sigma} V+[U, V]=0 .
$$

Parallel transport along this flat connection is defined by the path-ordered exponent

$$
\Omega_{C}(\gamma)=\mathcal{P} \exp \int_{\mathcal{C}}(U d \sigma+V d \tau)
$$

where $\mathcal{C}$ is some contour in $\mathbf{R}^{2}$. Restricting to transport along the contour defined by $\tau=\tau_{0}$ and $0 \leq \sigma \leq 2 \pi$ yields a monodromy matrix:

$$
T(2 \pi, \gamma)=\mathcal{P} \exp \int_{0}^{2 \pi} d \sigma U
$$

The flatness condition in eqn. (3.9) admits an infinite number of conservation laws, which translates to the fact that the trace of the monodromy matrix yields an infinite tower of local, mutually commuting charges $\widehat{Q}_{n}^{S^{5}}$ when expanded in powers of the spectral index about the poles of $U(\gamma= \pm 1$, in this case $):^{2}$

$$
\operatorname{tr} T(2 \pi, \gamma)=\sum_{n} \gamma^{n} \widehat{Q}_{n}^{S^{5}}
$$

The first nonvanishing charge $\widehat{Q}_{2}^{S^{5}}$, for example, is the Hamiltonian of the theory (on the $S^{5}$ subspace).

Moving beyond leading order in the semiclassical expansion, the essential difference between the $O(6)$ sigma model defined in eqn. (3.1) and the string action given in eqn. (2.6) is, as noted above, that worldsheet indices are contracted in the latter case with a nonflat worldsheet metric. Keeping the components of $h^{a b}$ explicit, the lightcone Hamiltonian derived from the string sigma model in eqn. (2.6) appears at leading order as

$$
H_{\mathrm{LC}}^{S^{5}}=-\frac{1}{2 p_{-} R^{2}}\left[h^{\tau \tau}\left(p_{-}^{2} y^{2}+y^{\prime 2}+\dot{y}^{2}\right)+2 h^{\tau \sigma} \dot{y} \cdot y^{\prime}\right]+O\left(1 / R^{4}\right),
$$

\footnotetext{
${ }^{2}$ In general, an expansion around some $\gamma$ that is finitely displaced from a singularity of $U$ will yield combinations of local and non-local quantities. One is of course free to redefine $\gamma$ such that the expansion about $\gamma=0$ in eqn. (3.12) is local.
} 
where $h^{\tau \tau}=-1+\tilde{h}^{\tau \tau} / R^{2}$ and $h^{\tau \sigma}=\tilde{h}^{\tau \sigma} / R^{2}$. The prescription will be to find a perturbation to the $(U, V)$-connection such that the Hamiltonian in eqn. (3.13) emerges in an appropriate limit from the charge $\widehat{Q}_{2}^{S_{5}}$ defined by eqn. (3.12). Such a perturbation is achieved by transforming the $U$ matrix according to

$$
U \rightarrow U=\frac{1}{1+\gamma}\left(1+u_{-} / R^{2}\right) A_{-}-\frac{1}{1-\gamma}\left(1+u_{+} / R^{2}\right) A_{+},
$$

where $u_{ \pm}$are given by

$$
u_{ \pm} \equiv-\frac{1}{2} \tilde{h}^{\tau \tau} \mp \frac{1}{3} \tilde{h}^{\tau \sigma}
$$

These perturbations should be treated as constants, to be replaced in the end with dynamical variables by fixing conformal gauge according to eqn. (2.12). The remaining quartic perturbations to the pp-wave theory will be naturally encoded in the semiclassical expansion of the underlying $O(6)$ (likewise, $O(4,2)$ ) sigma model. The matrix $V$ can be transformed in a similar way:

$$
V \rightarrow V=-\frac{1}{1+\gamma}\left(1+v_{-} / R^{2}\right) A_{-}-\frac{1}{1-\gamma}\left(1+v_{-} / R^{2}\right) A_{+},
$$

where $v_{ \pm}$may be chosen such that the perturbed Lax pair satisfies the flatness condition in eqn. (3.9). Given that the intent is simply to determine whether the higher local charges generated by the perturbed monodromy matrix are conserved when quantum fluctuations are included, fixing $V$ to satisfy the flatness condition is not really necessary: the complicated formulas for $v_{ \pm}$that do satisfy eqn. (3.9) will therefore not be needed.

The perturbation in eqn. (3.14) can be obtained by a slightly different method. When the path-ordered exponent defining the monodromy matrix is expanded, it can be seen that all odd products of the Lax matrix $U$ will not contribute to the final expression. By replacing all even products of $U$ according to the rule

$$
\begin{aligned}
U\left(\sigma_{1}\right) U\left(\sigma_{2}\right) \rightarrow & \frac{1}{\left(\gamma^{2}-1\right)^{2}}\left[h^{\sigma \sigma} A_{\sigma}\left(\sigma_{1}\right) A_{\sigma}\left(\sigma_{2}\right)-\gamma^{2} h^{\sigma \tau} A_{\sigma}\left(\sigma_{1}\right) A_{\tau}\left(\sigma_{2}\right)\right. \\
& \left.-\gamma^{2} h^{\tau \sigma} A_{\tau}\left(\sigma_{1}\right) A_{\sigma}\left(\sigma_{2}\right)-\gamma^{2} h^{\tau \tau} A_{\tau}\left(\sigma_{1}\right) A_{\tau}\left(\sigma_{2}\right)\right]
\end{aligned}
$$

the Hamiltonian in eqn. (3.13) is again obtained at leading order in the expansion. Computationally, this latter method seems to be much more efficient, and we will use eqn. (3.17) in what follows. At leading order in the $1 / R$ expansion, the first nonvanishing integral of motion descending from the monodromy matrix is thereby found to be

$$
Q_{2}^{S^{5}}=\frac{4 \pi}{R^{2}} \int_{0}^{2 \pi} d \sigma\left[h^{\tau \tau}\left(p_{-}^{2} y^{2}+y^{\prime 2}+\dot{y}^{2}\right)+2 h^{\tau \sigma} \dot{y} \cdot y^{\prime}\right]+O\left(1 / R^{4}\right)
$$

which, by construction, matches the desired structure in eqn. (3.13). 
The same construction may be carried out for the $A d S_{5}$ system. In fact, to make matters simple, we may borrow the Lax structure of the $O(6)$ model defined in eqns. (3.3) 3.7), replacing the $O(6)$ coordinates in eqn. (3.2) with the following Euclideanized $O(4,2)$ complex embedding coordinates:

$$
\mathcal{Z}_{1}=Z_{1}+i Z_{2} \quad \mathcal{Z}_{2}=Z_{3}+i Z_{4} \quad \mathcal{Z}_{3}=i Z_{0}-Z_{5}
$$

In this case, however, the Lax matrix $S_{A d S_{5}}$ will obey $S_{A d S_{5}}^{\dagger} S_{A d S_{5}}=-1$. Otherwise, the analysis above applies to the $A d S_{5}$ sector by direct analogy: expanding the perturbed $O(4,2)$ monodromy matrix in the spectral parameter yields a set of charges labeled by $\widehat{Q}_{n}^{A d S_{5}}$. The local charges for the entire theory are then given by

$$
\widehat{Q}_{n} \equiv \widehat{Q}_{n}^{S^{5}}-\widehat{Q}_{n}^{A d S_{5}}
$$

The corresponding currents will be labeled by $\mathcal{Q}_{n}$.

It turns out that the expansion in the spectral parameter $\gamma$ is arranged such that the path-ordered exponent defining the monodromy matrix can be computed explicitly to a given order in $\gamma$ by evaluating only a finite number of worldsheet integrals. The procedure for extracting local, canonically quantized currents is then completely analogous to that followed in computing the lightcone Hamiltonian described above. All gauge fixing is done after the currents are evaluated, all occurrences of $x^{-}$are replaced with dynamical variables by solving the conformal gauge constraints, and worldsheet metric corrections $\tilde{h}^{a b}$ are evaluated according to eqns. (2.15) above. We note, however, that previous studies involving the matching of integrable structures between gauge and string theory have found it necessary to invoke certain redefinitions of $\gamma$ to obtain agreement [7, 27]. It would be straightforward to allow for rather general redefinitions of the spectral parameter in the present calculation. When we turn to computing spectra and comparing with gauge theory, however, such redefinitions can lead to unwanted ambiguity. We will therefore be primarily interested in finding ratios of eigenvalue coefficients for which arbitrary redefinitions of $\gamma$ are irrelevant, and for simplicity we will simply retain the original definition of $\gamma$ given by eqn. (3.7) above.

As previously noted, the first current $\mathcal{Q}_{1}$ defined by eqn. (3.12) vanishes. In fact, all $\mathcal{Q}_{n}$ vanish for odd values of $n$, and this property of the integrable structure is mirrored on the gauge theory side. The first nonvanishing current emerging from the monodromy matrix is given by

$$
\begin{aligned}
\mathcal{Q}_{2}= & \frac{4 \pi}{R^{2}}\left(\left(\dot{x}^{A}\right)^{2}+\left(x^{\prime A}\right)^{2}+p_{-}^{2}\left(x^{A}\right)^{2}\right) \\
& +\frac{\pi}{R^{4}}\left\{2 z^{2}\left[y^{\prime 2}+2 z^{\prime 2}-\dot{y}^{2}\right]-2 y^{2}\left[z^{\prime 2}+2 y^{\prime 2}-\dot{z}^{2}\right]-\frac{4}{p_{-}^{2}}\left(\dot{x}^{A} x^{\prime A}\right)^{2}\right. \\
& \left.+\frac{1}{p_{-}^{2}}\left[3\left(\dot{x}^{A}\right)^{2}-\left(x^{\prime A}\right)^{2}\right]\left[\left(\dot{x}^{A}\right)^{2}+\left(x^{\prime A}\right)^{2}\right]+p_{-}^{2}\left[\left(x^{A}\right)^{2}\right]^{2}\right\}+O\left(1 / R^{6}\right) .
\end{aligned}
$$

The leading-order term is the quadratic pp-wave Hamiltonian, as expected, and the perturbation is strictly quartic in field fluctuations. All occurrences of $x^{-}$and all curvature 
corrections to the worldsheet metric $\tilde{h}^{a b}$ have been replaced with physical variables as described above. The final step is to express eqn. (3.21) in terms of canonically conjugate variables determined by directly varying the Lagrangian in eqn. (2.4). We obtain

$$
\begin{aligned}
& \mathcal{Q}_{2}= \frac{4 \pi}{R^{2}}\left(p_{-}^{2}\left(x^{A}\right)^{2}+\left(p_{A}\right)^{2}+\left(x^{\prime}\right)^{2}\right) \\
&+\frac{\pi}{R^{4}}\left\{2\left[-y^{2}\left(p_{z}^{2}+z^{\prime 2}+2 y^{\prime 2}\right)+z^{2}\left(p_{y}^{2}+y^{\prime 2}+2 z^{\prime 2}\right)\right]+p_{-}^{2}\left[\left(x^{A}\right)^{2}\right]^{2}\right. \\
&\left.-\frac{1}{p_{-}^{2}}\left\{\left[\left(p_{A}\right)^{2}\right]^{2}+2\left(p_{A}\right)^{2}\left(x^{\prime A}\right)^{2}+\left[\left(x^{\prime}\right)^{2}\right]^{2}\right\}+\frac{4}{p_{-}^{2}}\left(x^{\prime A} p_{A}\right)^{2}\right\}+O\left(1 / R^{6}\right) .
\end{aligned}
$$

Comparing this with eqn. (2.16) above, we see that, to the order of interest,

$$
\mathcal{Q}_{2}=8 \pi p_{-} H_{\mathrm{LC}} \text {. }
$$

As expected, the perturbed monodromy matrix precisely reproduces the structure of the lightcone Hamiltonian to quartic order in the semiclassical expansion. (Note that $\mathcal{Q}_{2}$ is only expected to be identified with the lightcone Hamiltonian up to an overall constant.)

Computationally, the expansion of the monodromy matrix becomes increasingly time consuming at higher orders in the spectral index. The situation can be mitigated to some extent by projecting the theory onto $A d S_{5}$ or $S^{5}$ excitations, eliminating all interaction terms from the quartic perturbation that mix fluctuations from both subspaces. We will eventually want to compute eigenvalue spectra in the block-diagonal subsectors discussed above (which require such a projection), so this maneuver will not affect the outcome.

The next nonvanishing $S^{5}$ current in the series is given by

$$
\begin{aligned}
\mathcal{Q}_{4}^{S^{5}}= & \frac{8 \pi}{3 R^{2}}\left(3-\pi^{2} p_{-}^{2}\right)\left(p_{-}^{2} y^{2}+p_{y}^{2}+y^{\prime 2}\right) \\
& +\frac{2 \pi}{3 p_{-}^{2} R^{4}}\left\{-3\left(p_{y}^{2}-2 p_{y} \cdot y^{\prime}+y^{\prime 2}\right)\left(p_{y}^{2}+2 p_{y} \cdot y^{\prime}+y^{\prime 2}\right)\right. \\
& -\pi^{2} p_{-}^{2}\left[4\left(p_{y} \cdot y^{\prime}\right)^{2}+\left(p_{y}^{2}+y^{\prime 2}\right)^{2}\right]-12 p_{-}^{2} y^{\prime 2} y^{2} \\
& \left.-p_{-}^{4} y^{2}\left[4 \pi^{2} p_{y}^{2}-3 y^{2}\right]-3 \pi^{2} p_{-}^{6}\left(y^{2}\right)^{2}\right\}+O\left(1 / R^{6}\right) .
\end{aligned}
$$

Although the quadratic interaction of $\mathcal{Q}_{4}^{S^{5}}$ is proportional to the pp-wave Hamiltonian on the $S^{5}$, the structure of the perturbing quartic interaction differs from that obtained for $\mathcal{Q}_{2}$. The corresponding $A d S_{5}$ current takes the form

$$
\begin{aligned}
\mathcal{Q}_{4}^{A d S_{5}}= & \frac{8 \pi}{3 R^{2}}\left(3-\pi^{2} p_{-}^{2}\right)\left(p_{-}^{2} z^{2}+p_{z}^{2}+z^{\prime 2}\right) \\
& +\frac{2 \pi}{3 p_{-}^{2} R^{4}}\left\{-3\left(p_{z}^{2}-2 p_{z} \cdot z^{\prime}+{z^{\prime}}^{2}\right)\left(p_{z}^{2}+2 p_{z} \cdot z^{\prime}+z^{\prime 2}\right)\right. \\
& -\pi^{2} p_{-}^{2}\left[4\left(p_{z} \cdot z^{\prime}\right)^{2}+\left(p_{z}^{2}+z^{\prime 2}\right)^{2}\right]+12 p_{-}^{2} z^{\prime 2} z^{2} \\
& \left.+p_{-}^{4} z^{2}\left[-4 \pi^{2} z^{\prime 2}+3 z^{2}\right]+\pi^{2} p_{-}^{6}\left(z^{2}\right)^{2}\right\}+O\left(1 / R^{6}\right)
\end{aligned}
$$


where the quadratic sector is again proportional to the pp-wave Hamiltonian, projected in this case onto the $A d S_{5}$ subspace. Continuing on to sixth-order in the spectral index, we find the $S^{5}$ current

$$
\begin{aligned}
\mathcal{Q}_{6}^{S^{5}}= & \frac{1}{15 R^{2}}\left\{4 \pi\left[45-40 \pi^{2} p_{-}^{2}+2 \pi^{4} p_{-}^{4}\right]\left(p_{-}^{2} y^{2}+p_{y}^{2}+y^{\prime 2}\right)\right\} \\
& +\frac{\pi}{15 p_{-}^{2} R^{4}}\left\{-45\left(p_{y}^{2}-2 p_{y} \cdot y^{\prime}+y^{\prime 2}\right)\left(p_{y}^{2}+2 p_{y} \cdot y^{\prime}+y^{\prime 2}\right)\right. \\
& -20 p_{-}^{2}\left[2 \pi^{2}\left(4\left(p_{y} \cdot y^{\prime}\right)^{2}+\left(p_{y}^{2}+y^{\prime 2}\right)^{2}\right)+9 y^{\prime 2} y^{2}\right] \\
& +p_{-}^{4}\left[2 \pi^{4}\left(4\left(p_{y} \cdot y^{\prime}\right)^{2}+3\left(p_{y}^{2}+y^{\prime 2}\right)^{2}\right)-160 \pi^{2} p_{y}^{2} y^{2}+45\left(y^{2}\right)^{2}\right] \\
+ & \left.8 \pi^{2} p_{-}^{6} y^{2}\left[\left(2 \pi^{2} p_{y}^{2}+\pi^{2} y^{\prime 2}\right)-15 y^{2}\right]+10 \pi^{4} p_{-}^{8}\left(y^{2}\right)^{2}\right\}+O\left(1 / R^{6}\right) .
\end{aligned}
$$

The quadratic piece of $\mathcal{Q}_{6}^{S^{5}}$ is again identical in structure to the pp-wave Hamiltonian. The analogous current in the $A d S_{5}$ subspace is arranged in a similar fashion:

$$
\begin{aligned}
\mathcal{Q}_{6}^{A d S_{5}}= & \frac{1}{15 R^{2}}\left\{4 \pi\left[45-40 \pi^{2} p_{-}^{2}+2 \pi^{4} p_{-}^{4}\right]\left(p_{-}^{2} z^{2}+p_{z}^{2}+z^{\prime 2}\right)\right\} \\
& +\frac{\pi}{15 p_{-}^{2} R^{4}}\left\{-45\left(p_{z}^{2}-2 p_{z} \cdot z^{\prime}+z^{\prime 2}\right)\left(p_{z}^{2}+2 p_{z} \cdot z^{\prime}+z^{\prime 2}\right)\right. \\
& -20 p_{-}^{2}\left[2 \pi^{2}\left(4\left(p_{z} \cdot z^{\prime}\right)^{2}+\left(p_{z}^{2}+z^{\prime 2}\right)^{2}\right)-9 z^{\prime 2} z^{2}\right] \\
& +p_{-}^{4}\left[2 \pi^{4}\left(4\left(p_{z} \cdot z^{\prime}\right)^{2}+3\left(p_{z}^{2}+z^{\prime 2}\right)^{2}\right)-160 \pi^{2} z^{\prime 2} z^{2}+45\left(z^{2}\right)^{2}\right] \\
+ & \left.8 \pi^{2} p_{-}^{6} y^{2}\left(\pi^{2} z^{\prime 2}+5 z^{2}\right)-6 \pi^{4} p_{-}^{8}\left(z^{2}\right)^{2}\right\}+O\left(1 / R^{6}\right) .
\end{aligned}
$$

While we will not present explicit formulas for the resulting currents, it is easy to carry this out to eighth order in $\gamma$.

Taken separately, each current can be viewed as a free pp-wave Hamiltonian plus a quartic interaction. This is particularly useful, as it allows us to quantize each charge exactly at leading order and express the perturbation in terms of free pp-wave oscillators. More explicitly, we quantize the quadratic sectors of these currents by expanding the fluctuation fields in their usual Fourier components:

$$
\begin{aligned}
x^{A}(\sigma, \tau) & =\sum_{n=-\infty}^{\infty} x_{n}^{A}(\tau) e^{-i k_{n} \sigma}, \\
x_{n}^{A}(\tau) & =\frac{i}{\sqrt{2 \omega_{n}}}\left(a_{n}^{A} e^{-i \omega_{n} \tau}+a_{n}^{A \dagger} e^{i \omega_{n} \tau}\right) .
\end{aligned}
$$

The quadratic (pp-wave) equations of motion

$$
\ddot{x}^{A}-x^{\prime \prime}+p_{-}^{2} x^{A}=0
$$


are satisfied by setting $k_{n}=n$ (integer), and $\omega_{n}=\sqrt{p_{-}^{2}+k_{n}^{2}}$, where the operators $a_{n}^{A}$ and $a_{n}^{A \dagger}$ obey the commutation relation $\left[a_{m}^{A}, a_{n}^{B \dagger}\right]=\delta_{m n} \delta^{A B}$.

In accordance with integrability, we expect that the local charges in eqns. (3.22 3.27) should all be mutually commuting. Expressed in terms of quantum raising and lowering operators, we can check the commutators of the hidden local charges directly. To avoid mixing issues, We will need to select out closed subsectors of each charge which completely decouple from the remaining terms in the theory. We have already noted that the Hamiltonian $\widehat{Q}_{2}$ is known to be closed under $A d S_{5}$ and $S^{5}$ string states forming symmetric-traceless irreps in their spacetime indices. The equivalent gauge theory statement is that the dilatation generator is closed in certain $\mathfrak{s l}(2)$ and $\mathfrak{s u}(2)$ projections. Since the complete tower of corresponding charges in the gauge theory (including the dilatation generator) can be diagonalized by a single set of $\mathfrak{s l}(2)$ or $\mathfrak{s u}(2)$ Bethe equations, it is a reasonable guess that the full tower of local string charges decouples under corresponding projections. (A similar conjecture is made, for example, in [23, 27].) Following the treatment in [31, we therefore define the following $\operatorname{Ad}_{5}$ oscillators

$$
a_{n}=\frac{1}{\sqrt{2}}\left(a_{n}^{j}+i a_{n}^{k}\right) \quad \bar{a}_{n}=\frac{1}{\sqrt{2}}\left(a_{n}^{j}-i a_{n}^{k}\right) \quad(j \neq k),
$$

which satisfy the standard relations

$$
\left[a_{n}, a_{m}^{\dagger}\right]=\left[\bar{a}_{n}, \bar{a}_{m}^{\dagger}\right]=\delta_{n m} \quad\left[a_{n}, \bar{a}_{m}^{\dagger}\right]=\left[\bar{a}_{n}, a_{m}^{\dagger}\right]=0 .
$$

When restricted to these oscillators, the symmetric-traceless projection in the $A d S_{5}$ subspace is achieved by setting all $\bar{a}_{n}, \bar{a}_{n}^{\dagger}$ to zero (see [31] for details). A corresponding definition on the $S^{5}$ takes the form

$$
a_{n}=\frac{1}{\sqrt{2}}\left(a_{n}^{j^{\prime}}+i a_{n}^{k^{\prime}}\right) \quad \bar{a}_{n}=\frac{1}{\sqrt{2}}\left(a_{n}^{j^{\prime}}-i a_{n}^{k^{\prime}}\right) \quad\left(j^{\prime} \neq k^{\prime}\right)
$$

where the symmetric-traceless projection is again invoked by setting $\bar{a}_{n}, \bar{a}_{n}^{\dagger}$ to zero. In other words, we can test the commutativity of the local charges in the $A d S_{5}$ and $S^{5}$ symmetrictraceless projections by rewriting their oscillator expansions according to eqns. (3.30 3.32) and setting all $\bar{a}_{n}, \bar{a}_{n}^{\dagger}$ to zero.

Since the currents are expanded to $O\left(1 / R^{4}\right)$, we only require that the commutators vanish to $O\left(1 / R^{6}\right)$. This simplifies the problem somewhat, since we only need to compute commutators involving at most six oscillators. On the subspace of symmetric-traceless $A d S_{5}$ string states, we obtain

$$
\left[\widehat{Q}_{n}^{A d S_{5}}, \widehat{Q}_{m}^{A d S_{5}}\right]=O\left(1 / R^{6}\right) \quad n, m \in 2, \ldots, 8 .
$$

The corresponding projection on the $S^{5}$ yields

$$
\left[\widehat{Q}_{n}^{S^{5}}, \widehat{Q}_{m}^{S^{5}}\right]=O\left(1 / R^{6}\right) \quad n, m \in 2, \ldots, 8 .
$$

We therefore find evidence for the existence of a tower of mutually commuting charges (within these particular closed sectors) that are conserved perturbatively by the quantized theory. 


\section{Spectral comparison with gauge theory}

Given the freedom involved in redefinitions of the spectral parameter, it may seem that any spectral agreement between the string charges computed above and corresponding quantities in the gauge theory would be rather arbitrary. We therefore seek a comparison of integrable structures on both sides of the duality that avoids this ambiguity. It turns out that such a test is indeed possible in the symmetric-traceless sector of $S^{5}$ excitations, which will map in the gauge theory to the closed $\mathfrak{s u}(2)$ sector. We will further restrict ourselves to computing spectra associated with the following two-impurity string states:

$$
a_{q}^{j^{\prime} \dagger} a_{-q}^{k^{\prime} \dagger}|J\rangle
$$

The analysis for three or higher-impurity states would require an accounting of interactions between $A d S_{5}$ and $S^{5}$ string excitations; as noted above, however, this dramatically complicates the computational analysis. (We intend to return to the question of higher-impurity string integrability in a future study.) The ground state $|J\rangle$ is understood to carry $J$ units of angular momentum on the $S^{5}$, and the two-impurity $S O(4)$ subspace above comprises a $16 \times 16$-dimensional sub-block of the Hamiltonian. In addition, the mode indices (labeled here by $q$ ) of physical string states must sum to zero to satisfy the usual level-matching condition (the Virasoro constraint is understood to be satisfied by the leading-order solution to the equations of motion; any higher-order information contained in the $T_{01}$ component of eqn. (2.12) is irrelevant).

To simplify the analysis, and for comparison with [31, 36, 37, 38, we will also rescale each of the charges computed above by a factor of $R^{2}$ :

$$
\widehat{Q}_{n} \rightarrow R^{2} \widehat{Q}_{n}
$$

The two-impurity matrix elements of the charge $\widehat{Q}_{2}^{S^{5}}$ are then given by (note that the index notation is chosen here to align with [31, 36, 37, 38]):

$$
\begin{aligned}
\left\langle J\left|a_{q}^{a^{\prime}} a_{-q}^{b^{\prime}}\left(\widehat{Q}_{2}^{S^{5}}\right) a_{-q}^{c^{\prime} \dagger} a_{q}^{d^{\prime} \dagger}\right| J\right\rangle & =16 \pi \omega_{q} \delta^{a^{\prime} d^{\prime}} \delta^{b^{\prime} c^{\prime}} \\
& -\frac{8 \pi q^{2}}{J \sqrt{\lambda^{\prime}} \omega_{q}^{2}}\left[\left(3+2 q^{2} \lambda\right) \delta^{a^{\prime} d^{\prime}} \delta^{b^{\prime} c^{\prime}}-\delta^{a^{\prime} c^{\prime}} \delta^{b^{\prime} d^{\prime}}+\delta^{a^{\prime} b^{\prime}} \delta^{c^{\prime} d^{\prime}}\right]+O\left(1 / J^{2}\right)
\end{aligned}
$$

where the upper spacetime indices $a^{\prime}, b^{\prime}, c^{\prime}, \ldots$ label the transverse $S O(4)$ directions in the $S^{5}$ subspace. The radius $R$ has been replaced with the angular momentum $J$, and $p_{-}$has been replaced with $1 / \sqrt{\lambda^{\prime}}$ via

$$
J / p_{-}=R^{2}=\sqrt{\lambda}
$$

As expected, contributions to the pp-wave limit of eqn. (4.2) all lie on the diagonal. Up to an overall factor, one may further check that the correction terms at $O(1 / J)$ agree with 
those computed in [36, 37, projected onto the $S^{5}$ subspace. The next higher charges in the series yield matrix elements given by

$$
\begin{aligned}
& \left\langle J\left|a_{q}^{a^{\prime}} a_{-q}^{b^{\prime}}\left(\widehat{Q}_{4}^{S^{5}}\right) a_{-q}^{c^{\prime} \dagger} a_{q}^{d^{\prime} \dagger}\right| J\right\rangle=\frac{32 \pi \omega_{q}}{3 \lambda^{\prime}}\left(3 \lambda^{\prime}-\pi^{2}\right) \delta^{a^{\prime} d^{\prime}} \delta^{b^{\prime} c^{\prime}} \\
& -\frac{16 \pi}{3 \lambda^{5 / 2} \omega_{q}^{2} J}\left\{\left[\pi^{2}\left(2+q^{2} \lambda^{\prime}\right)+3 q^{2} \lambda^{\prime 2}\left(3+2 q^{2} \lambda^{\prime}\right)\right] \delta^{a^{\prime} d^{\prime}} \delta^{b^{\prime} c^{\prime}}\right. \\
& +\left[\pi^{2}\left(2+q^{2} \lambda^{\prime}\right)-3 q^{2} \lambda^{\prime 2}\right] \delta^{a^{\prime} c^{\prime}} \delta^{b^{\prime} d^{\prime}} \\
& \left.+q^{2} \lambda^{\prime}\left(3 \lambda^{\prime}-\pi^{2}\right) \delta^{a^{\prime} b^{\prime}} \delta^{c^{\prime} d^{\prime}}\right\}+O\left(1 / J^{2}\right), \\
& \left\langle J\left|a_{q}^{a^{\prime}} a_{-q}^{b^{\prime}}\left(\widehat{Q}_{6}^{S^{5}}\right) a_{-q}^{c^{\prime} \dagger} a_{q}^{d^{\prime} \dagger}\right| J\right\rangle=\frac{16 \pi}{15 \omega_{q} \lambda^{\prime 2}}\left(2 \pi^{4}-40 \pi^{2} \lambda^{\prime}+45 \lambda^{\prime 2}\right) \omega_{q}^{2} \delta^{a^{\prime} d^{\prime}} \delta^{b^{\prime} c^{\prime}} \\
& +\frac{8 \pi}{15 \lambda^{\prime 7 / 2} \omega_{q}^{2}}\left\{\left[2 \pi^{4}\left(4+q^{2} \lambda^{\prime}\left(5+q^{2} \lambda^{\prime}\right)\right)-40 \pi^{2} \lambda^{\prime}\left(2+q^{2} \lambda^{\prime}\right)\right.\right. \\
& \left.-45 q^{2} \lambda^{\prime 3}\left(3+2 q^{2} \lambda^{\prime}\right)\right] \delta^{a^{\prime} d^{\prime}} \delta^{b^{\prime} c^{\prime}}+\left[2 \pi^{4}\left(4+q^{2} \lambda^{\prime}\right)-40 \pi^{2} \lambda^{\prime}\left(2+q^{2} \lambda^{\prime}\right)\right. \\
& \left.\left.+45 q^{2} \lambda^{\prime 3}\right] \delta^{a^{\prime} c^{\prime}} \delta^{b^{\prime} d^{\prime}}+q^{2} \lambda^{\prime}\left[\lambda^{\prime}\left(40 \pi^{2}-45 \lambda^{\prime}\right)-2 \pi^{4}\right] \delta^{a^{\prime} b^{\prime}} \delta^{c^{\prime} d^{\prime}}\right\}+O\left(1 / J^{2}\right) \text {. }
\end{aligned}
$$

We will again project onto symmetric-traceless irreps of $S O(4) \times S O(4)$, transforming as $(\mathbf{1}, \mathbf{1} ; \mathbf{3}, \mathbf{3})$ in an $S U(2)^{2} \times S U(2)^{2}$ notation. Although it is not necessarily guaranteed that the symmetric-traceless states will diagonalize the higher charges $\widehat{Q}_{4}$ and $\widehat{Q}_{6}$ at quartic order, this can be checked directly at one-loop order in $\lambda^{\prime}$ by computing the eigenvectors of the charges above (the higher-loop version of this check is much more difficult because the above charges are no longer completely block diagonal under the $S O(4)$ projection, a fact that can be seen in the structure of $\mathcal{Q}_{2}$ above). The $\widehat{Q}_{2}^{S^{5}}$ eigenvalue between symmetric-traceless $(\mathbf{1}, \mathbf{1} ; \mathbf{3}, \mathbf{3}) S^{5}$ states (denoted by $Q_{2}^{S^{5}}$ ) is then found to be

$$
Q_{2}^{S^{5}}=16 \pi\left(\omega_{q}-\frac{q^{2} \sqrt{\lambda^{\prime}}}{J}\right)+O\left(1 / J^{2}\right)
$$

Up to an overall constant, this is just the two-impurity energy shift originally reported in [36, 37]. The corresponding eigenvalues of the higher charges $\widehat{Q}_{4}$ and $\widehat{Q}_{6}$ can be computed 
in an analogous fashion:

$$
\begin{aligned}
Q_{4}^{S^{5}}= & \frac{32 \pi}{3}\left\{\frac{\omega_{q}}{\lambda^{\prime}}\left[3 \lambda^{\prime}-\pi^{2}\right]\right. \\
& \left.-\frac{\pi}{\lambda^{\prime 5 / 2} \omega_{q}^{2} J}\left[\pi^{2}\left(2+q^{2} \lambda^{\prime}\right)+3 q^{2} \lambda^{\prime 3} \omega_{q}^{2}\right]\right\}+O\left(1 / J^{2}\right), \\
Q_{6}^{S^{5}}= & \frac{16 \pi}{15}\left\{\frac{\omega_{q}}{\lambda^{\prime 2}}\left(2 \pi^{4}-40 \pi^{2} \lambda^{\prime}+45 \lambda^{\prime 2}\right)-\frac{1}{\omega_{q}^{2} \lambda^{\prime / 2} J}\left[40 \pi^{2} \lambda^{\prime}\left(2+q^{2} \lambda^{\prime}\right)\right.\right. \\
& \left.\left.+45 q^{2} \lambda^{\prime 4} \omega_{q}^{2}-2 \pi^{4}\left(4+q^{2} \lambda^{\prime}\left(3+q^{2} \lambda^{\prime}\right)\right)\right]\right\}+O\left(1 / J^{2}\right) .
\end{aligned}
$$

Similar formulas can be extracted for the $A d S_{5}$ charges $Q_{2}^{A d S_{5}}, Q_{4}^{A d S_{5}}$ and $Q_{6}^{A d S_{5}}$, which are diagonalized by symmetric-traceless $(\mathbf{3}, \mathbf{3} ; \mathbf{1}, \mathbf{1})$ string states excited in the $A d S_{5}$ subspace. Though we have not given explicit formulas, it is also straightforward to obtain the corresponding eigenvalues for $Q_{8}^{A d S_{5}}$ and $Q_{8}^{S^{5}}$.

By modifying the Inozemtsev spin chain of [6] to exhibit higher-loop BMN scaling, Beisert, Dippel and Staudacher were able to formulate a long-range Bethe ansatz for the gauge theory in the closed $\mathfrak{s u}(2)$ sector [7] (we will simply state their results here, referring the reader to [7] for further details). In essence, the Bethe ansatz encodes the interactions of pseudoparticle excitations on a spin lattice and, in terms of pseudoparticle momenta $p_{k}$, the ansatz given in [7] diagonalizes the entire tower of local gauge theory $\mathfrak{s u}(2)$ charges. The eigenvalues of these charges, which we label here as $D_{n}$, are given by

$$
D_{n}=\sum_{k=1}^{M} q_{n}\left(p_{k}\right), \quad q_{n}(p)=\frac{2 \sin \left(\frac{p}{2}(n-1)\right)}{n-1}\left(\frac{\sqrt{1+8 g^{2} \sin ^{2}(p / 2)}-1}{2 g^{2} \sin ^{2}(p / 2)}\right)^{n-1}
$$

where $g^{2} \equiv \lambda / 8 \pi^{2}$, and the index $k$ runs over the total number $M$ of pseudoparticle excitations (or $R$-charge impurities) on the spin lattice. These eigenvalues can then be expanded perturbatively in inverse powers of the gauge theory $R$-charge $\mathcal{R}$ by approximating the pseudoparticle momenta $p_{k}$ by the expansion

$$
p_{k}=\sum_{j} \frac{f_{j}\left(n_{k}\right)}{\mathcal{R}^{j / 2}}
$$

where $f_{j}$ are functions of the integer mode numbers $n_{k}$, determined by solving the Bethe equations explicitly to a given order in $1 / \mathcal{R}$.

In general, we wish to identify the local string charges with linear combinations of corresponding charges in the gauge theory. From eqn. (4.8), however, it is easy to see that as one moves up the ladder of higher charges in the gauge theory, the eigenvalues $D_{n}$ of these charges have leading contributions at higher and higher powers of $g^{2} / \mathcal{R}^{2}$ in the large- $\mathcal{R}$, small- $\lambda$ double-scaling expansion. This is puzzling because the string eigenvalues computed 
above do not exhibit similar properties. The difference in scaling behavior therefore motivates the following prescription for identifying the eigenvalues of the higher local charges on both sides of the correspondence:

$$
Q_{n}-N=C\left(\frac{n}{2} D_{n}\right)^{2 / n}
$$

$N$ here counts the number of string worldsheet impurities and $C$ is an arbitrary constant. Fractional powers of the gauge theory charges $D_{n}$ are well defined in terms of the doublescaling expansion, so that the right-hand side of eqn. (4.10) is in fact just a linear combination of conserved quantities in the gauge theory.

A potential subtlety arises when matching $Q_{n}$ and $D_{n}$ in this fashion for $n>2$ beyond one-loop order in $\lambda$. The problem is that, under the identification in eqn. (4.10), information from string energy eigenvalues at $O\left(1 / J^{2}\right)$ and higher is required to completely characterize the higher-loop (in $\lambda$ ) coefficients of the gauge theory charges $D_{n}$. The essential reason for this is that the string loop expansion is in powers of the modified 't Hooft coupling which, in terms of the gauge theory $R$-charge $\mathcal{R}$, is

$$
\lambda^{\prime}=\lambda / J^{2}=\lambda / \mathcal{R}^{2}
$$

In other words, under eqn. (4.10), it is impossible to disentangle higher-order $1 / J$ contributions to the string charges $Q_{n}$ from higher-order $\lambda$ corrections to $D_{n}$. The prescription given in eqn. (4.10) therefore holds only to one-loop order in $\lambda$, where knowing the $1 / J$ corrections in the string theory is sufficient.

Furthermore, since the local charges in the string and gauge theories are only identified up to an overall multiplicative constant, directly comparing the spectra of each theory is not especially rigorous. A convenient quantity to work with, however, is the ratio of the $O(1 / J)$ eigenvalue correction to the pp-wave coefficient: at first loop order in $\lambda$ this ratio eliminates all ambiguity associated with overall constants and $\gamma$ redefinitions, and thus provides a meaningful comparison with gauge theory. (The analogous quantity computed for charges in the $A d S_{5}$ subspace is not free from such ambiguities.) We therefore arrange the one-loop, two-impurity eigenvalues of local $S^{5}$ string theory charges according to

$$
Q_{n}^{S^{5}}=2+q^{2} \lambda^{\prime}\left(\Lambda_{n, 0}+\frac{\Lambda_{n, 1}}{J}\right)+O\left(\lambda^{2}\right)+O\left(1 / J^{2}\right)
$$

where the numbers $\Lambda_{n, 0}$ and $\Lambda_{n, 1}$ characterize eigenvalue coefficients in the pp-wave limit and at $O(1 / J)$, respectively, and $q$ is the mode number associated with the two-impurity string states defined above. On the gauge theory side we make a similar arrangement:

$$
\left(\frac{n}{2} D_{n}\right)^{2 / n}=\frac{q^{2} \lambda}{\mathcal{R}^{2}}\left(\bar{\Lambda}_{n, 0}+\frac{\bar{\Lambda}_{n, 1}}{\mathcal{R}}\right)+O\left(\lambda^{2}\right)+O\left(1 / \mathcal{R}^{4}\right)
$$

where the integer $q$ is a mode number associated with the momenta of pseudoparticle excitations on the spin lattice (which, in turn, correspond to roots of the $\mathfrak{s u}(2)$ Bethe equations). 


\begin{tabular}{|c|cc|}
\hline$n$ & $\Lambda_{n, 1} / \Lambda_{n, 0}$ & $\bar{\Lambda}_{n, 1} / \bar{\Lambda}_{n, 0}$ \\
\hline 2 & -2 & -2 \\
3 & 0 & 0 \\
4 & -2 & -2 \\
5 & 0 & 0 \\
6 & -2 & -2 \\
7 & 0 & 0 \\
8 & -2 & -2 \\
\hline
\end{tabular}

Table 1: Ratios of $O(1 / J)$ (or $O(1 / \mathcal{R})$ ) corrections to pp-wave/BMN coefficients in string and gauge theory local charges.

The $R$-charge $\mathcal{R}$ is understood to be identified with the string angular momentum $J$ via eqn. (4.11).

The quantities $\Lambda_{2,0}$ and $\Lambda_{2,1}$ for the string Hamiltonian $Q_{2}$ can be computed from the eigenvalue formula in eqn. (4.6) (or, alternatively, retrieved from the two-impurity string results reported in [36, 37]). We find the following ratio:

$$
\Lambda_{2,1} / \Lambda_{2,0}=-2
$$

As shown in [36, 37, this agrees with the corresponding gauge theory prediction at one-loop order in $\lambda$ :

$$
\bar{\Lambda}_{2,1} / \bar{\Lambda}_{2,0}=-2
$$

The ratio of $O(1 / J)$ eigenvalue corrections to pp-wave coefficients is in fact -2 for all of the nonvanishing string charges. Under the matching prescription in eqn. (4.10), this agrees with the gauge theory perfectly. (The odd charges vanish altogether on both sides of the correspondence.) We summarize the results of this comparison for the first eight charges in the series in Table 1. It would be satisfying to test this agreement at higher loop-orders in $\lambda$. The corresponding computation at two-loop order, however, would require evaluating the local string theory charges at $O\left(1 / R^{6}\right)$ in the semiclassical expansion, where several subtleties of perturbation theory (and, for that matter, lightcone quantization) would need to be addressed. This emphasizes the need to understand the quantum string theory at higher orders in the expansion away from the pp-wave limit.

\section{Discussion}

We have provided evidence that an infinite tower of local, mutually commuting bosonic charges of type IIB string theory on $A d S_{5} \times S^{5}$, known to exist in the classical theory, can 
also be identified in the quantum theory. In addition, we have provided a prescription for matching certain eigenvalues of these charges in a protected subsector of the string theory to corresponding eigenvalues in the closed $\mathfrak{s u}(2)$ sector of the gauge theory. The fact that the spectra of local string charges computed here can only be matched to corresponding quantities in the gauge theory via the matching prescription in eqn. (4.10), however, indicates that the monodromy matrix used to derive the local string charges is substantially different from that which would give rise to the proposed quantum string Bethe ansatz of [30] (or, since they are equivalent at one-loop order, the corresponding $\mathfrak{s u}(2)$ Bethe ansatz in the gauge theory). In other words, we expect that there is a Lax representation for the string sigma model that gives rise to hidden local charges that can be compared directly with the gauge theory, without having to take fractional powers or linear combinations.

There are a number of additional tests of integrability in the quantum string theory which, in the context of the present calculation, should be relatively straightforward. By computing the quartic interactions among fluctuations in the $A d S_{5}$ and $S^{5}$ subspaces for each of the higher local charges studied here, it would be easy, for example, to find the resulting spectra of three- or higher-impurity string states. Apart from the difficulty of actually computing the mixing interactions, this would provide a simple check on the methodology employed here. A more difficult problem would be to address whether the integrable structure of the string theory respects supersymmetry. By formulating a supersymmetric Lax representation that generates the complete interaction Hamiltonian computed in [36, 37], one might be able to show that each of the higher local charges are individually supersymmetric, and a comparison with gauge theory could be carried out in the closed $\mathfrak{s u}(1 \mid 1)$ sector studied in [31, 38, (the corresponding sector of the string theory would be comprised of symmetrized fermionic excitations in the $(\mathbf{3}, \mathbf{1} ; \mathbf{3}, \mathbf{1})$ or $(\mathbf{1}, \mathbf{3} ; \mathbf{1}, \mathbf{3})$ of $S O(4) \times S O(4))$.

Ultimately, the hope is that the arsenal of techniques associated with integrable systems will lead to an exact solution to the string formulation of large- $N_{c}$ Yang-Mills theory. Alternatively, a proof that both sides of the duality are diagonalized by identical Bethe equations should be obtainable. At present, the major obstacle preventing such a proof is the disagreement between gauge and string theory at three-loop order in the 't Hooft coupling. The fact that the integrable systems of both theories seem to agree in certain limited cases, however, stands as strong evidence that they are likely to be equivalent.

\section{Acknowledgments}

We would like to thank Curtis Callan for guidance and for reading the manuscript. We also thank Sergey Frolov, Vladimir Kazakov, Tristan McLoughlin, Andrei Mikhailov, John Schwarz and Arkady Tseytlin for many helpful discussions, The Ohio State University and the Institute for Advanced Study for hospitality, and the James A. Cullen Memorial Fund for support. This work was supported in part by US Department of Energy grant DE-FG0392-ER40701. 


\section{References}

[1] D. Berenstein, J. M. Maldacena and H. Nastase, "Strings in flat space and pp waves from $\mathcal{N}=4$ super Yang Mills," JHEP 0204, 013 (2002) [arXiv:hep-th/0202021].

[2] R. R. Metsaev, "Type IIB Green-Schwarz superstring in plane wave Ramond-Ramond background," Nucl. Phys. B 625, 70 (2002) [arXiv:hep-th/0112044].

[3] R. R. Metsaev and A. A. Tseytlin, "Exactly solvable model of superstring in plane wave Ramond-Ramond background," Phys. Rev. D 65, 126004 (2002) [arXiv:hepth/0202109].

[4] J. A. Minahan and K. Zarembo, "The Bethe-ansatz for $\mathcal{N}=4$ super Yang-Mills," JHEP 0303, 013 (2003) [arXiv:hep-th/0212208].

[5] N. Beisert and M. Staudacher, "The $\mathcal{N}=4$ SYM integrable super spin chain," Nucl. Phys. B 670, 439 (2003) [arXiv:hep-th/0307042].

[6] D. Serban and M. Staudacher, "Planar $\mathcal{N}=4$ gauge theory and the Inozemtsev long range spin chain," arXiv:hep-th/0401057.

[7] N. Beisert, V. Dippel and M. Staudacher, "A novel long range spin chain and planar $\mathcal{N}=4$ super Yang-Mills," arXiv:hep-th/0405001.

[8] N. Beisert, C. Kristjansen and M. Staudacher, "The dilatation operator of $\mathcal{N}=4$ super Yang-Mills theory," Nucl. Phys. B 664, 131 (2003) [arXiv:hep-th/0303060].

[9] N. Beisert, "The complete one-loop dilatation operator of $\mathcal{N}=4$ super Yang-Mills theory," Nucl. Phys. B 676, 3 (2004) [arXiv:hep-th/0307015].

[10] C. G. Callan, Jr., J. Heckman, T. McLoughlin and I. Swanson, "Lattice Super YangMills: A Virial Approach to Operator Dimensions," Nucl. Phys. B 701, 180 (2004) [arXiv:hep-th/0407096].

[11] N. Beisert, "The dilatation operator of $\mathcal{N}=4$ super Yang-Mills theory and integrability," arXiv:hep-th/0407277.

[12] S. S. Gubser, I. R. Klebanov and A. M. Polyakov, "A semi-classical limit of the gauge/string correspondence," Nucl. Phys. B 636, 99 (2002) [arXiv:hep-th/0204051].

[13] S. Frolov and A. A. Tseytlin, "Semiclassical quantization of rotating superstring in $A d S_{5} \times S^{5}, "$ JHEP 0206, 007 (2002) [arXiv:hep-th/0204226].

[14] S. Frolov and A. A. Tseytlin, "Multi-spin string solutions in $A d S_{5} \times S^{5}$," Nucl. Phys. B 668, 77 (2003) [arXiv:hep-th/0304255].

[15] A. A. Tseytlin, "Spinning strings and AdS/CFT duality," arXiv:hep-th/0311139. 
[16] I. Bena, J. Polchinski and R. Roiban, "Hidden symmetries of the $A d S_{5} \times S^{5}$ superstring," Phys. Rev. D 69, 046002 (2004) [arXiv:hep-th/0305116].

[17] L. F. Alday, "Nonlocal charges on $A d S_{5} \times S^{5}$ and pp-waves," JHEP 0312, 033 (2003) [arXiv:hep-th/0310146].

[18] L. Dolan, C. R. Nappi and E. Witten, "Yangian symmetry in $D=4$ superconformal Yang-Mills theory," arXiv:hep-th/0401243.

[19] L. Dolan, C. R. Nappi and E. Witten, "A relation between approaches to integrability in superconformal Yang-Mills theory," JHEP 0310, 017 (2003) [arXiv:hep-th/0308089].

[20] L. D. Faddeev, "How Algebraic Bethe Ansatz works for integrable model," arXiv:hepth/9605187.

[21] L. D. Faddeev and L. A. Takhtajan, "Hamiltonian Methods In The Theory Of Solitons," Berlin, Germany: Springer (1987) 592 p. (Springer Series in Soviet Mathematics).

[22] G. Mandal, N. V. Suryanarayana and S. R. Wadia, "Aspects of semiclassical strings in $A d S_{5}, "$ Phys. Lett. B 543, 81 (2002) [arXiv:hep-th/0206103].

[23] G. Arutyunov and M. Staudacher, "Matching higher conserved charges for strings and spins," JHEP 0403, 004 (2004) [arXiv:hep-th/0310182].

[24] G. Arutyunov, S. Frolov, J. Russo and A. A. Tseytlin, "Spinning strings in $A d S_{5} \times S^{5}$ and integrable systems," Nucl. Phys. B 671, 3 (2003) [arXiv:hep-th/0307191].

[25] G. Arutyunov, J. Russo and A. A. Tseytlin, "Spinning strings in $A d S_{5} \times S^{5}$ : New integrable system relations," Phys. Rev. D 69, 086009 (2004) [arXiv:hep-th/0311004].

[26] J. Engquist, "Higher conserved charges and integrability for spinning strings in $A d S_{5} \times$ $S^{5}, " J H E P$ 0404, 002 (2004) [arXiv:hep-th/0402092].

[27] V. A. Kazakov, A. Marshakov, J. A. Minahan and K. Zarembo, "Classical / quantum integrability in AdS/CFT," JHEP 0405, 024 (2004) [arXiv:hep-th/0402207].

[28] V. A. Kazakov and K. Zarembo, "Classical/quantum integrability in non-compact sector of AdS/CFT," arXiv:hep-th/0410105.

[29] N. Beisert, V. A. Kazakov and K. Sakai, "Algebraic Curve for the $S O(6)$ sector of AdS/CFT," arXiv:hep-th/0410253.

[30] G. Arutyunov, S. Frolov and M. Staudacher, "Bethe ansatz for quantum strings," arXiv:hep-th/0406256.

[31] T. McLoughlin and I. Swanson, "N-impurity superstring spectra near the pp-wave limit," Nucl. Phys. B 702, 86 (2004) [arXiv:hep-th/0407240]. 
[32] S. S. Gubser, I. R. Klebanov and A. M. Polyakov, "Gauge theory correlators from non-critical string theory," Phys. Lett. B 428, 105 (1998) [arXiv:hep-th/9802109].

[33] N. Beisert, "Spin chain for quantum strings," arXiv:hep-th/0409054.

[34] I. Swanson, "On the integrability of string theory in $A d S_{5} \times S^{5}$," arXiv:hep-th/0405172.

[35] A. Parnachev and A. V. Ryzhov, "Strings in the near plane wave background and AdS/CFT," JHEP 0210, 066 (2002) [arXiv:hep-th/0208010].

[36] C. G. Callan, Jr., H. K. Lee, T. McLoughlin, J. H. Schwarz, I. Swanson and X. Wu, "Quantizing string theory in $A d S_{5} \times S^{5}$ : Beyond the pp-wave," Nucl. Phys. B 673, 3 (2003) [arXiv:hep-th/0307032].

[37] C. G. Callan, Jr., T. McLoughlin and I. Swanson, "Holography beyond the Penrose limit," Nucl. Phys. B 694, 115 (2004) [arXiv:hep-th/0404007].

[38] C. G. Callan, Jr., T. McLoughlin and I. Swanson, "Higher impurity AdS/CFT correspondence in the near-BMN limit," Nucl. Phys. B 700, 271 (2004) [arXiv:hepth/0405153].

[39] R. Kallosh, J. Rahmfeld and A. Rajaraman, "Near horizon superspace," JHEP 9809, 002 (1998) [arXiv:hep-th/9805217].

[40] R. Kallosh and A. A. Tseytlin, "Simplifying superstring action on $A d S_{5} \times S^{5}$," JHEP 9810, 016 (1998) [arXiv:hep-th/9808088].

[41] R. R. Metsaev and A. A. Tseytlin, "Type IIB superstring action in $A d S_{5} \times S^{5}$ background," Nucl. Phys. B 533, 109 (1998) [arXiv:hep-th/9805028].

[42] R. R. Metsaev, "Light cone gauge formulation of IIB supergravity in $A d S_{5} \times S^{5}$ background and AdS/CFT correspondence," Phys. Lett. B 468, 65 (1999) [arXiv:hepth/9908114].

[43] R. R. Metsaev and A. A. Tseytlin, "Superstring action in $A d S_{5} \times S^{5}$ : kappa-symmetry light cone gauge," Phys. Rev. D 63, 046002 (2001) [arXiv:hep-th/0007036].

[44] N. Beisert, "Higher loops, integrability and the near BMN limit," JHEP 0309, 062 (2003) [arXiv:hep-th/0308074].

[45] N. Beisert, "The su(2|3) dynamic spin chain," Nucl. Phys. B 682, 487 (2004) [arXiv:hepth/0310252].

[46] K. Pohlmeyer, "Integrable Hamiltonian Systems And Interactions Through Quadratic Constraints," Commun. Math. Phys. 46, 207 (1976). 